\title{
Differential microRNA expression profiles determined by next-generation sequencing in three fulvestrant-resistant human breast cancer cell lines
}

\author{
JUAN GUO*, KELI HE* , HUI ZENG* ${ }^{*}$, YU SHI, PENG YE, QIAN ZHOU, ZHONGYA PAN and XINGHUA LONG \\ Department of Laboratory Medicine, Zhongnan Hospital of Wuhan University, Wuhan, Hubei 430071, P.R. China
}

Received February 7, 2018; Accepted February 6, 2019

DOI: $10.3892 / \mathrm{ol} .2019 .10061$

\begin{abstract}
Fulvestrant resistance is a major clinical issue in the treatment of endocrine-based breast cancer. MicroRNAs (miRNAs) are known to serve an important role in tumor chemoresistance. In the present study, the association between miRNA expression profiles and fulvestrant resistance was investigated in human breast cancer cell lines. Three fulvestrant-resistant breast cancer cell lines, namely MCF-7-CC, MCF-7-TT and MCF-7-21, were established using the human breast cancer cell line MCF-7 as the parental cell line and fulvestrant as the screening drug in vitro. Next-generation sequencing was used to determine the miRNA expression profiles in these cell lines. Subsequently, Gene Ontology (GO) and Kyoto Encyclopedia of Genes and Genomes (KEGG) pathway analyses were performed to determine the biological functions of differentially expressed miRNAs. In total, 1,536 miRNAs were detected in all the samples, including 1,240 known miRNAs and 296 predicted miRNAs. It was observed that the differential miRNA expression profiles varied among the three fulvestrant-resistant cell lines (MCF-7-CC, MCF-7-TT and MCF-7-21), and certain differentially expressed miRNAs were only detected in one or two of the cell lines. A total of 257 miRNAs that were differentially expressed between MCF-7-CC and MCF-7 cells were detected, among which 69 miRNAs were upregulated and 188 miRNAs were downregulated. In addition, 270 miRNAs with significantly different expression between MCF-7-TT and MCF-7 cells were observed, including 180 upregulated and 90 downregulated miRNAs. Between MCF-7-21 and MCF-7 cells, a total of 227 miRNAs were differentially expressed, among which 52 miRNAs were upregulated and 175 miRNAs were
\end{abstract}

Correspondence to: Dr Xinghua Long, Department of Laboratory Medicine, Zhongnan Hospital of Wuhan University, 169 Donghu Road, Wuhan, Hubei 430071, P.R. China

E-mail: zhoulongxinghua@qq.com

*Contributed equally

Key words: microRNA, breast cancer, MCF-7, fulvestrant, resistance downregulated. The miRNAs that were differentially expressed in the three fulvestrant-resistant cell lines as compared with the parental MCF-7 cell line were primarily involved in the following biological processes: Biological regulation, extracellular matrix-receptor interaction, the Notch signaling pathway and focal adhesion. Taken together, the results suggested that miR-143, miR-145, miR-137, miR-424 and miR-21 may serve important roles in fulvestrant resistance in breast cancer. The study findings may provide a basis for further research on the treatment of fulvestrant-resistant breast cancer.

\section{Introduction}

Breast cancer is one of the most common types of tumor in women, and has been estimated to account for $29 \%$ of all novel cases of cancer diagnosed among American women in 2016 (1). Breast cancer is an endocrine-dependent cancer, with $\sim 70 \%$ of cases being estrogen receptor positive $\left(\mathrm{ER}^{+}\right)$. Estrogen has been reported to stimulate tumor growth, and endocrine therapy is one of the main treatment strategies for $\mathrm{ER}^{+}$breast cancer patients, decreasing the estrogen levels or inhibiting the function of estrogen. Effective endocrine therapy is important for the treatment of breast cancer, and reduces the rate of relapse and mortality of patients.

Fulvestrant, a selective ER downregulator (SERD), is specifically used to treat postmenopausal breast cancer patients following the failure of first-line endocrine therapies (such as tamoxifen). SERDs function by binding to and blocking the ER, thus leading to the inhibition of estrogen signaling by targeting the ER (2). However, drug resistance is a factor that limits the efficacy of fulvestrant. Certain breast cancer patients acquire resistance against fulvestrant as a result of long-term treatment through various mechanisms, including glycoprotein 88 overexpression (3), functional interaction of human epidermal growth factor receptor 2 (HER2)/neu with HER3 (4), and methylation of the ER promoter region (5). It is crucial to understand the underlying mechanisms involved in fulvestrant resistance, since it is a major clinical obstacle in breast cancer treatment; however, these mechanisms remain unknown.

Aberrant expression of microRNAs (miRNAs) is considered to be one of the numerous mechanisms of drug resistance (6). miRNAs are a class of small non-coding RNAs (with a length of 22 nucleotides) that regulate gene expression at the post-transcriptional level by binding to the 
3'-untranslated region (3'UTR) of target mRNAs to either inhibit mRNA translation or target the molecule for degradation. miRNAs participate in a series of important biological processes, including cell proliferation, differentiation and apoptosis, as well as signal transduction $(7,8)$. A number of studies in recent years have reported that aberrant expression of miRNA is closely associated with drug resistance in breast cancer, including fulvestrant resistance (9-11). However, the differential miRNA expression profiles of fulvestrant-resistant human breast cancer cell lines have rarely been studied. Thus, three different fulvestrant-resistant cell lines were established in the current study, which represent a variety of drug resistance conditions. These cells may exhibit exogenous or endogenous resistance-associated characteristics, as reported previously (12-16).

The current study aimed to investigate the association between differential miRNA expression profiles and fulvestrant resistance in human breast cancer cells. The known and predicted miRNAs were detected by next-generation sequencing, which has a stronger ability to identify genes compared with DNA microarray (17).

\section{Materials and methods}

Cells and reagents. The human breast cancer cell line MCF-7 was purchased from the American Type Culture Collection (ATCC; Manassas, VA, USA) and cultured in Dulbecco's modified Eagle's medium (DMEM; HyClone; GE Healthcare Life Sciences, Logan, UT, USA) supplemented with $10 \%$ fetal bovine serum (FBS; HyClone; GE Healthcare Life Sciences) at $37^{\circ} \mathrm{C}$ in a humidified atmosphere containing 5\% $\mathrm{CO}_{2}$. Fulvestrant was purchased from Sigma-Aldrich (Merck KGaA, Darmstadt, Germany).

\section{Establishment of three fulvestrant-resistant cell lines}

Establishment of MCF-7-CC cell line. An MCF-7-CC cell line was established using the human breast cancer cell line MCF-7, which served as the parental cell line. Resistance was induced using fulvestrant as the screening drug by stepwise induction, starting with a low concentration $(12.5 \mathrm{nM})$ of fulvestrant (13). This method was conducted in two sequential phases: Adaptation and consolidation. In the adaptation phase, MCF-7 cells were cultured in complete medium until the adhesion rate reached $70 \%$; next, $12.5 \mathrm{nM}$ fulvestrant was added to cells plated in DMEM (containing phenol red) with 5\% FBS for $72 \mathrm{~h}$. Apoptotic cells were then removed and the complete medium was replaced until the viable cells reached a $70 \%$ adherence rate. This process was repeated twice, and MCF-7 cells were collectively incubated with $12.5 \mathrm{nM}$ fulvestrant for a total of three times. Subsequently, the treated cells were incubated with $25,50,100,200,400,800$ and 1,00 $0 \mathrm{nM}$ fulvestrant, respectively, in the same manner. Previous studies $(14,15)$ reported using $100 \mathrm{nM}$ fulvestrant to establish the fulvestrant-resistant cell line in medium containing charcoal-stripped FBS. However, medium containing FBS was used in the current study; thus, it was necessary to increase the fulvestrant concentration, and $1,000 \mathrm{nM}$ was used as the final dose. In the consolidation phase, cells incubated with $1,000 \mathrm{nM}$ fulvestrant in DMEM were then treated with $1,200 \mathrm{nM}$ fulvestrant for an additional 10 times until normal growth was observed, indicating that the MCF-7-CC cell line was successfully established.

Establishment of MCF-7-TT cell line. An MCF-7-TT cell line was established by stepwise temporal induction, beginning with a high concentration of fulvestrant (13). The process of induction was also divided into two stages, including adaptation and consolidation. Briefly, MCF-7 cells were cultured in complete medium until the adhesion rate reached $70 \%$. Next, the complete medium (DMEM with 10\% FBS) was discarded, and the screening medium [DMEM (containing phenol red) with 5\% FBS] with 1,000 nM fulvestrant was used to induce the cells for $1 \mathrm{~h}$. The screening medium was discarded and replaced with complete culture medium subsequent to washing three times with PBS (Hyclone; GE Healthcare Life Sciences). Apoptotic cells were then removed and the complete medium was replaced until the viable cells reached a 70\% adherence rate. This process was repeated twice, and the MCF-7 cells were collectively induced with $1,000 \mathrm{nM}$ fulvestrant for $1 \mathrm{~h}$ for a total of three times. Subsequently, 2, 4, 8, 12, 24, 36 and $48 \mathrm{~h}$ of induction were sequentially performed with $1,000 \mathrm{nM}$ fulvestrant following the aforementioned screening procedure. In the consolidation phase, the cell line obtained from the adaptation stage was cultured in 1,000 $\mathrm{nM}$ fulvestrant containing medium for $72 \mathrm{~h}$ for a total of ten times, following which the MCF-7-TT cell line was successfully established.

Establishment of MCF-7-21 cell line. An MCF-7-21 cell line was established using the human breast cancer cell line MCF-7 as the parental cell line, which was incubated with a high concentration of fulvestrant for 21 days (16). MCF-7 cells were cultured in the DMEM (containing phenol red) with 5\% FBS, and then 1,000 nM fulvestrant was added to the medium. MCF-7 cells were cultured for 21 days, following which the MCF-7-21 cell line was successfully established.

Total cellular RNA extraction. Total RNA of the three fulvestrant-resistant cell lines was extracted using TRIzol reagent (Invitrogen; Thermo Fisher Scientific, Inc., Waltham, MA, USA) according to the manufacturer's protocol. A NanoDrop spectrophotometer (Thermo Fisher Scientific, Inc.), Qubit 2.0 fluorometer (Thermo Fisher Scientific, Inc.) and an Agilent 2100 bioanalyzer (Agilent Technologies, Inc., Santa Clara, CA, USA) were used to detect the purity, concentration and integrity of RNA samples, respectively.

Small RNA library construction and sequencing. miRNA high-throughput sequencing was performed by Beijing Biomarker Biotechnology Co., Ltd. (Beijing, China), and the small RNA library was constructed and sequenced as follows: A total of $1.5 \mu \mathrm{g}$ RNA was used as the initial sample, and the volume was made up to $6 \mu 1$ with water. The library was constructed using the small RNA Sample Prep kit (Illumina, Inc., San Diego, CA, USA) following the manufacturer's recommendations. As a phosphate group was present at the 5 ' terminus and a hydroxyl group at the $3^{\prime}$ terminus, small RNAs were ligated at the $3^{\prime}$ and $5^{\prime}$ ends using T4 RNA Ligase 1 and T4 RNA Ligase 2 (truncated), respectively. Next, cDNA was synthesized by reverse transcription (RT) with a RevertAid First Strand cDNA Synthesis kit (Thermo Fisher 
Table I. Sequences of primers using for reverse transcription-quantitative polymerase chain reaction.

\begin{tabular}{lll}
\hline Target & Forward primer $\left(5^{\prime}-3^{\prime}\right)$ & Reverse primer $\left(5^{\prime}-3^{\prime}\right)$ \\
\hline U6 & CTCGCTTCGGCAGCACA & AACGCTTCACGAATTTGCGT \\
miR-582-3p & TAACTGGTTGAACAAC & GTGCAGGGTCCGAGGT \\
miR-143-5p & GGTGCAGTGCTGCATC & GTGCAGGGTCCGAGGT \\
miR-145-5p & GTCCAGTTTTCCCAGGA & GTGCAGGGTCCGAGGT
\end{tabular}

miR, microRNA.

Scientific, Inc.), and the cDNA fragments were subsequently amplified by polymerase chain reaction (PCR), cloned and sequenced. The target gene fragment was isolated by a gel separation technique, cut and collected as a small RNA library. Following the generation of the library, the concentration of the constructed library was detected by Qubit 2.0 device, and the library was diluted to a concentration of $1 \mathrm{ng} / \mu \mathrm{l}$. The insert size was then tested using the Agilent 2100 bioanalyzer and the working concentration of the library was determined by a quantitative PCR (qPCR) method to obtain a high-quality library. High-throughput sequencing of the library was finally performed on the Illumina HiSeq 2500 sequencing platform (Illumina, Inc.).

miRNA identification and differential expression analysis. MiRDeep2 software was used to identify known miRNAs and predict novel miRNAs (18). The expression of miRNAs in each sample was analyzed statistically, and the expression level was normalized using a transcript per million algorithm (19). The thresholds for significant miRNA differential expression analysis were set as follows: $\log _{2}$ fold change (FC)| of $\geq 1$ and false discovery rate (FDR) of $\leq 0.01$. The FC represents the ratio of expression between two samples. The significant P-value obtained from the hypothesis can be expressed as the probability of miRNAs expressing no difference between the fulvestrant-resistant cell line and MCF-7 cell line. As the differential expression analysis of miRNAs is an independent statistical hypothesis test for the expression of multiple miRNAs, false-positive results may be detected. Thus, the Benjamini-Hochberg correction method was used to test the P-value obtained from the hypothesis. Finally, the FDR was used as the key indicator of differential expression of miRNAs during the screening process (20). The genes obtained from the differential expression analysis were referred to as differentially expressed genes (DEG). Cluster analysis for differentially expressed miRNAs was performed using Heatmap Illustrator software (21).

Validation of three selected differentially expressed miRNAs by $R T-q P C R$. RT-qPCR was used to confirm the expression levels of three miRNAs, namely miR-582-3p, miR-143-5p and miR-145-5p. These miRNAs were obtained from the next-generation sequencing analysis and were differentially expressed in all three fulvestrant-resistant cell lines compared with the parental MCF-7 cell line. Briefly, the extracted total cellular RNA was reverse transcribed into cDNA using a miScript II Reverse Transcription kit (Qiagen
$\mathrm{GmbH}$, Hilden, Germany) according to the manufacturer's protocol. qPCR was then performed using a LightCycler ${ }^{\circledR}$ 480 II Real-time PCR instrument (Roche Diagnostics, Basel, Switzerland) with $10-\mu 1$ PCR reaction mixture, containing $1 \mu \mathrm{l} \mathrm{cDNA,} 5 \mu 1$ 2X LightCycler ${ }^{\circledR} 480$ SYBR Green I Master (Roche Diagnostics), $0.2 \mu \mathrm{l}$ universal primer (Qiagen $\mathrm{GmbH}$ ), $0.2 \mu \mathrm{l}$ miRNA-specific primer and $3.6 \mu \mathrm{l}$ nuclease-free water. The primer sequences are presented in Table I. U6 small nuclear RNA was used as the internal control for the normalization of miRNA expression. The reaction mixture was pre-incubated at $95^{\circ} \mathrm{C}$ for $10 \mathrm{~min}$, followed by 40 cycles of $95^{\circ} \mathrm{C}$ for $10 \mathrm{sec}$ and $60^{\circ} \mathrm{C}$ for $30 \mathrm{sec}$. Each sample was run in triplicate. The relative expression of the selected differentially expressed miRNAs was calculated using the $2^{-\Delta \Delta \mathrm{Cq}}$ method (22).

Gene Ontology (GO) and Kyoto Encyclopedia of Genes and Genomes (KEGG) pathway analyses of target genes. MicroRNA.org, a comprehensive resource of miRNA target predictions and expression profiles (23), and the RNAhybrid tool that is primarily used as a means for miRNA target prediction (24) were used to predict the DEGs. Wallenius' non-central hypergeometric distribution-based analysis of GO enrichment of the DEGs was implemented using the GOseq $\mathrm{R}$ packages (25). In addition, KOBAS software (26) was used to assess the statistical enrichment of DEGs in KEGG pathways.

Statistical analysis. SPSS version 22 (IBM Corp., Armonk, NY, USA) was used for statistical analysis. Differences were evaluated using one-way analysis of variance with a post-hoc Student-Newman-Keuls test. $\mathrm{P}<0.05$ was considered to indicate a statistically significant difference.

\section{Results}

Expression profile of miRNAs in fulvestrant-resistant cell lines. A next-generation sequencing instrument (Illumina HiSeq 2500) was used to detect the expression profile of differentially expressed miRNAs. The results revealed that 1,536 miRNAs were detected in all samples, including 1,240 known miRNAs and 296 predicted miRNAs. A total of 257 miRNAs with significant differences between MCF-7-CC and MCF-7 cells were identified $(\mathrm{P}<0.05)$, of which 69 miRNAs were upregulated $(\mathrm{P}<0.05)$, and 188 were downregulated $(\mathrm{P}<0.05)$. There were 270 differentially expressed miRNAs between MCF-7-TT and MCF-7 cells $(\mathrm{P}<0.05)$, of which 
Table II. Statistical data of differentially expressed miRNAs in fulvestrant-resistant cell lines as compared with the MCF-7 parental cell line.

Differentially expressed miRNAs

\begin{tabular}{lccr}
\cline { 2 - 4 } Cell lines & Total no. & Upregulated & Downregulated \\
\hline MCF-7 vs. MCF-7-CC & 257 & 69 & 188 \\
MCF-7 vs. MCF-7-TT & 270 & 180 & 90 \\
MCF-7 vs. MCF-7-21 & 227 & 52 & 175
\end{tabular}

miRNA, microRNA.

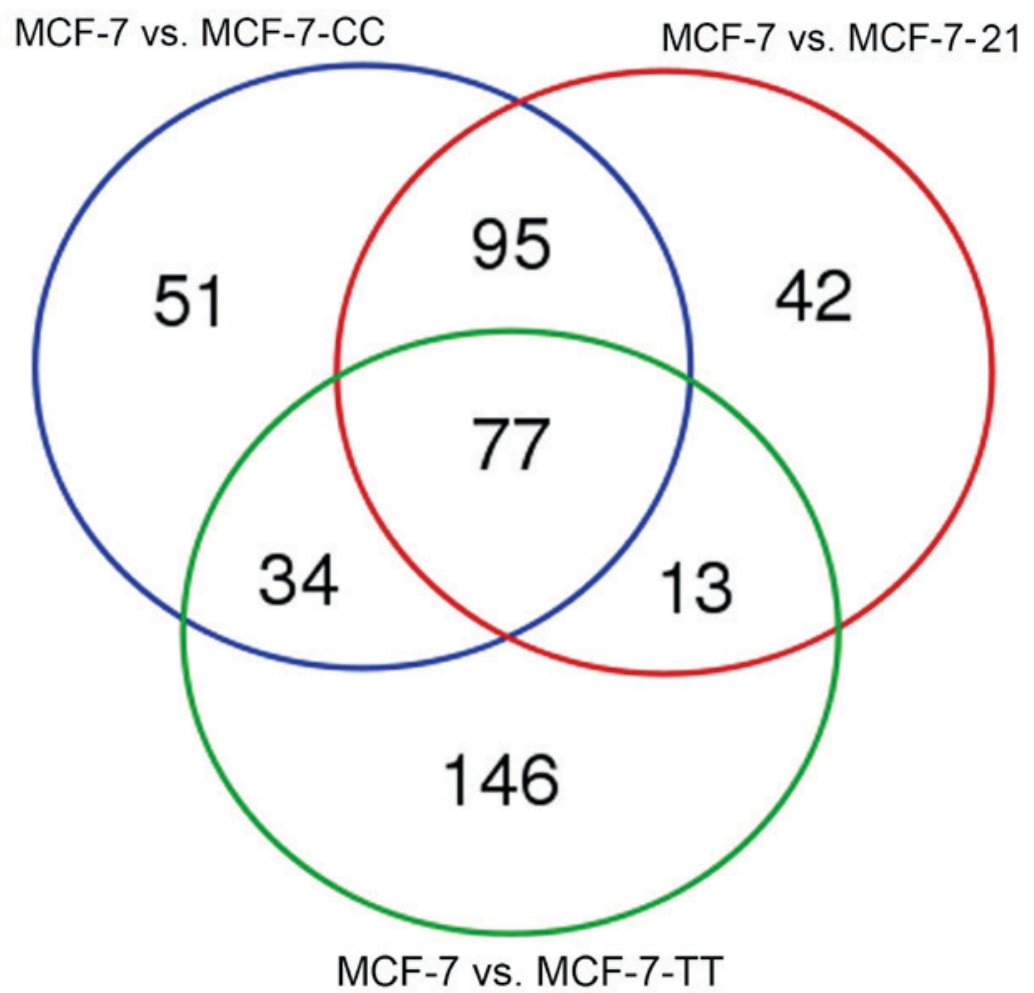

Figure 1. Overview of differentially expressed miRNAs in three fulvestrant-resistant cells compared with the MCF-7 parental cell line. A Venn diagram illustrates the overlapping results of the differentially expressed miRNAs in MCF-7-CC vs. MCF-7, MCF-7-TT vs. MCF-7, and MCF-7-21 vs. MCF-7 cells. Circles include the numbers of both upregulated and downregulated miRNAs of each pair-wise comparison. miRNA, microRNA.

180 miRNAs were upregulated $(\mathrm{P}<0.05)$ and 90 were downregulated $(\mathrm{P}<0.05)$. It was also observed that 227 miRNAs exhibited significantly different expression between MCF-7-21 and MCF-7 cells $(\mathrm{P}<0.05)$, of which 52 miRNAs were upregulated $(\mathrm{P}<0.05)$ and 175 miRNAs were downregulated $(\mathrm{P}<0.05)$. These results are listed in Table II.

The overlapping results of the differentially expressed miRNAs in MCF-7-CC vs. MCF-7, MCF-7-TT vs. MCF-7, and MCF-7-21 vs. MCF-7 cells are displayed in Fig. 1. In total, there were 77 common differentially expressed miRNAs across the three drug-resistant cell lines, including miR-1246, miR-143, miR-145, miR-424 and miR-137. Additionally, 42 miRNAs were differentially expressed only in MCF-7-21 vs. MCF-7 cells, including miR-188-5p, miR-4326 and miR-542, while 51 miRNAs were only differentially expressed in MCF-7-CC vs. MCF-7 cells, including let-7c-3p, miR-199b-3p and miR-210-3p. Furthermore, 146 miRNAs that were differentially expressed only in MCF-7-TT vs. MCF-7 cells were reported, including miR-101-3p, miR-141-5p and miR-15a. Overall, the results revealed that the differential miRNA expression profiles varied among the three fulvestrant-resistant cell lines, and that certain miRNAs were differentially expressed in all three MCF-7-CC, MCF-7-TT and MCF-7-21 cell lines; however, a number of miRNAs were only reported in one or two of the cell lines. The significantly upregulated or downregulated miRNAs in the three drug-resistant breast cancer cell lines are presented in Table III. miR-148a, miR-31, miR-21, miR-498 and miR-29b were significantly upregulated, while miR-143, miR-145, miR-424 and miR-137 were significantly downregulated in the fulvestrant-resistant cell lines. Subsequently, differentially expressed miRNAs with the same or similar expression profile were clustered by hierarchical 
Table III. Certain of the differentially expressed miRNA in the three fulvestrant-resistant breast cancer cell lines.

\begin{tabular}{|c|c|c|c|c|c|c|}
\hline \multirow[b]{2}{*}{ Cell lines } & \multicolumn{2}{|c|}{ Upregulated miRNAs } & \multirow[b]{2}{*}{ P-value } & \multicolumn{2}{|c|}{ Downregulated miRNAs } & \multirow[b]{2}{*}{ P-value } \\
\hline & miRNA & $\log _{2} \mathrm{FC}$ & & miRNA & $\log _{2} \mathrm{FC}$ & \\
\hline \multirow[t]{8}{*}{ MCF-7 vs. MCF-7-CC } & miR-148a-3p & 2.9113 & $\mathrm{P}<0.01$ & $\operatorname{miR}-137$ & -4.2516 & $\mathrm{P}<0.01$ \\
\hline & $\operatorname{miR}-31-3 p$ & 1.3064 & $\mathrm{P}<0.01$ & miR-143-5p & -5.4926 & $\mathrm{P}<0.01$ \\
\hline & $\operatorname{miR}-215-5 p$ & 3.3344 & $\mathrm{P}<0.01$ & miR-145-5p & -3.4729 & $\mathrm{P}<0.01$ \\
\hline & miR-489-5p & 24.5083 & $\mathrm{P}<0.01$ & $\operatorname{miR}-424-3 p$ & -2.807 & $\mathrm{P}<0.01$ \\
\hline & miR-182-5p & 1.5574 & $\mathrm{P}<0.01$ & miR-335-3p & -26.0166 & $\mathrm{P}<0.01$ \\
\hline & miR-1-3p & 2.0917 & $\mathrm{P}<0.01$ & miR-221-3p & -1.3765 & $\mathrm{P}<0.01$ \\
\hline & miR-498-3p & 24.5083 & $\mathrm{P}<0.01$ & $\operatorname{miR}-222-5 p$ & -1.221 & $\mathrm{P}<0.01$ \\
\hline & $\operatorname{miR}-582-3 p$ & 1.4994 & $\mathrm{P}<0.01$ & $\operatorname{miR}-26 b-3 p$ & -2.1457 & $\mathrm{P}<0.01$ \\
\hline \multirow[t]{8}{*}{ MCF-7 vs. MCF-7-TT } & miR-122-5p & 3.9638 & $\mathrm{P}<0.01$ & miR-137 & -2.5543 & $\mathrm{P}<0.01$ \\
\hline & $\operatorname{miR}-21-3 p$ & 1.5632 & $\mathrm{P}<0.01$ & miR-143-5p & -3.0583 & $\mathrm{P}<0.01$ \\
\hline & miR-148a-3p & 1.5609 & $\mathrm{P}<0.01$ & $\operatorname{miR}-145-5 p$ & -2.6236 & $\mathrm{P}<0.01$ \\
\hline & $\operatorname{miR}-422 \mathrm{a}$ & 2.3538 & $\mathrm{P}<0.01$ & $\operatorname{miR}-146 a-5 p$ & -5.0017 & $\mathrm{P}<0.01$ \\
\hline & $\operatorname{miR}-29 a-5 p$ & 1.1446 & $\mathrm{P}<0.01$ & miR-424-3p & -3.4994 & $\mathrm{P}<0.01$ \\
\hline & $\operatorname{miR}-31-3 p$ & 1.0498 & $\mathrm{P}<0.01$ & $\operatorname{miR}-504-5 p$ & -2.5182 & $\mathrm{P}<0.01$ \\
\hline & miR-629-5p & 2.0143 & $\mathrm{P}<0.01$ & miR-342-3p & -1.9604 & $\mathrm{P}<0.01$ \\
\hline & $\operatorname{miR}-582-3 p$ & 1.0648 & $\mathrm{P}<0.01$ & miR-935 & -2.3213 & $\mathrm{P}<0.01$ \\
\hline \multirow[t]{8}{*}{ MCF-7 vs. MCF-7-21 } & $\operatorname{miR}-148 a-3 p$ & 3.3875 & $\mathrm{P}<0.01$ & $\operatorname{miR}-137$ & -2.5542 & $\mathrm{P}<0.01$ \\
\hline & miR-182-5p & 1.6882 & $\mathrm{P}<0.01$ & miR-143-5p & -3.6957 & $\mathrm{P}<0.01$ \\
\hline & miR-99a-3p & 1.0275 & $\mathrm{P}<0.01$ & miR-145-5p & -1.8248 & $\mathrm{P}<0.01$ \\
\hline & miR-100-5p & 1.2957 & $\mathrm{P}<0.01$ & $\operatorname{miR}-221-3 p$ & -1.2638 & $\mathrm{P}<0.01$ \\
\hline & miR-29b-3p & 1.4432 & $\mathrm{P}<0.01$ & $\operatorname{miR}-222-5 p$ & -2.3876 & $\mathrm{P}<0.01$ \\
\hline & $\operatorname{miR}-215-5 p$ & 4.251 & $\mathrm{P}<0.01$ & $\operatorname{miR}-424-3 p$ & -1.072 & $\mathrm{P}<0.01$ \\
\hline & miR-182-5p & 1.6882 & $\mathrm{P}<0.01$ & miR-127-3p & -25.3999 & $\mathrm{P}<0.01$ \\
\hline & miR-582-3p & 2.3284 & $\mathrm{P}<0.01$ & miR-1276 & -3.828267 & $\mathrm{P}<0.01$ \\
\hline
\end{tabular}

miRNA/miR, microRNA.

cluster analysis. The clustering results of differently expressed miRNAs are shown as a heat map in Fig. 2.

$R T-q P C R$ validation of three selected differentially expressed miRNAs. RT-qPCR was used to validate the expression of three selected differentially expressed miRNAs, namely miR-582-3p, miR-143-5p and miR-145-5p, which were significantly differentially expressed in the three fulvestrant-resistant cell lines compared with the parental MCF-7 cell line (Fig. 3). This demonstrated that the expression profiles of these miRNAs detected by RT-qPCR were consistent with the result of next-generation sequencing.

GO and KEGG pathway analyses of differentially expressed miRNAs. To further understand the biological function of the predicted targets, GO and KEGG pathway enrichment analyses were performed. The results of GO enrichment analysis were similar in the three fulvestrant-resistant cell lines. The analysis of differential miRNA expression in MCF-7 vs. MCF-7-CC, MCF-7 vs. MCF-7-TT, and MCF-7 vs. MCF-7-21 cells are shown in Fig. 4A-C, respectively. GO annotation analysis included three categories, as follows: Biological process, cellular component and molecular function. The targets of the differentially expressed miRNAs were associated to the biological processes of biological regulation, metabolism, response to stimulus, hormone secretion and cell killing. The enriched cellular components were primarily associated with cell, organelle, membrane and cell junction. In addition, the main molecular functions were reported to be binding, catalytic activity, molecular transducer activity and chemoattractant activity.

Furthermore, the significant results of KEGG pathway enrichment analysis for MCF-7 vs. MCF-7-CC, MCF-7 vs. MCF-7-TT, and MCF-7 vs. MCF-7-21 cells are listed in Fig. 5A-C, respectively. The targets of the differentially expressed miRNAs in MCF-7 vs. MCF-7-CC cells were markedly enriched in the extracellular matrix (ECM)-receptor interaction, Notch signaling pathway, focal adhesion and ErbB signaling pathways. In MCF-7 vs. MCF-7-TT cells, the markedly enriched pathways included osteoclast differentiation, melanogenesis, basal cell carcinoma, glycosaminoglycan biosynthesis-chondroitin sulfate and ECM-receptor interaction. Finally, in MCF-7 vs. MCF-7-21 cells, the markedly enriched pathways included the ECM-receptor interaction, focal adhesion, glycosaminoglycan biosynthesis-chondroitin sulfate and Notch signaling. 


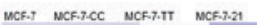

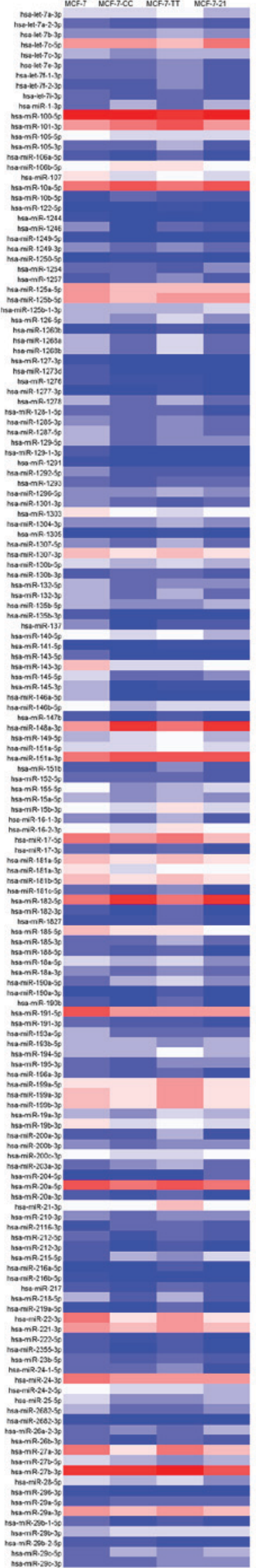

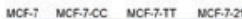

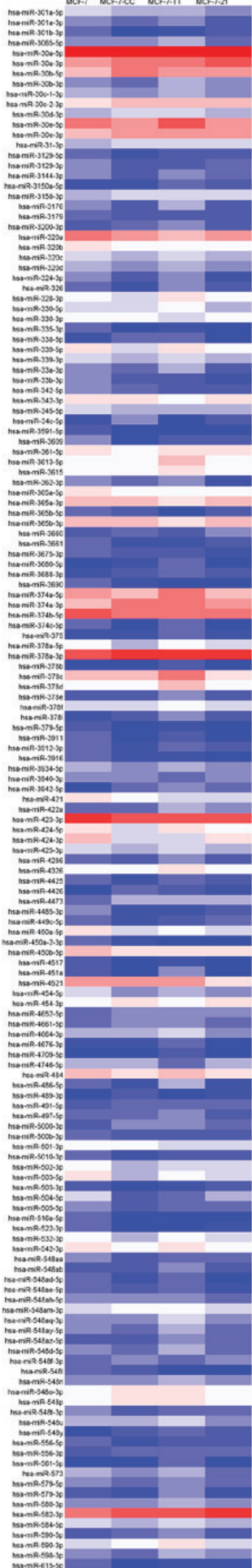

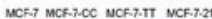

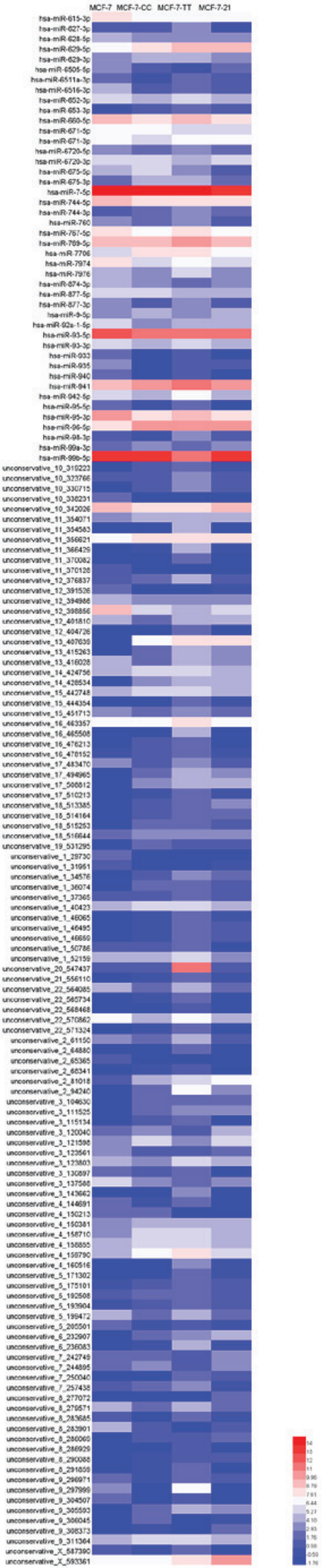

Figure 2. Hierarchical cluster of differentially expressed microRNAs in MCF-7, MCF-7-CC, MCF-7-TT and MCF-7-21 cell lines. The heatmap shows differentially expressed miRNA within a hierarchical cluster. Rows represent different miRNAs and columns represent different samples. The color scale illustrates the relative expression levels of miRNAs. Red indicates high relative expression levels of miRNAs, and blue indicates low relative expression levels of miRNAs. miRNA, microRNA. 


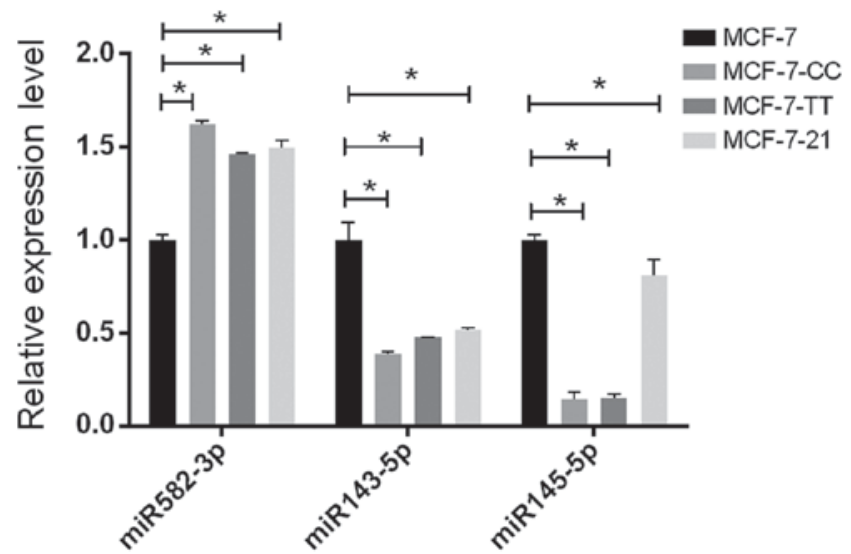

Figure 3. Validation of three selected differentially expressed miRNAs by reverse transcription-quantitative polymerase chain reaction. The relative expression levels of miR-582-3p, miR-143-5p and miR-145-5p in the MCF-7 and three fulvestrant-resistant cell lines are displayed. U6 small nuclear RNA was used as the internal control for the normalization of miRNA expression. ${ }^{*} \mathrm{P}<0.01$. miRNA/miR, microRNA.

\section{Discussion}

miRNAs regulate gene expression at the post-transcriptional level. Evidence suggests that miRNAs are often dysregulated in human tumors, and can function as oncogenes or tumor suppressors (27), serving important roles in the tumor progression. It has been reported that the aberrant expression of miRNA is associated with drug resistance (6). Understanding the targets of miRNAs and the underlying mechanism of drug resistance can contribute to new developments in the treatment of drug resistance in breast cancer.

In the present study, the differential expression of miRNAs in MCF-7 cells and three fulvestrant-resistant breast cancer cell lines was detected by next-generation sequencing. It was observed that the differential miRNA expression profiles were different among the three fulvestrant-resistant cell lines as compared with the parental MCF-7 cells, and certain miRNAs were only differentially expressed in only one or two of the cell lines. RT-qPCR was then performed to further validate the expression levels of miR-582-3p, miR-143-5p and miR-145-5p in MCF-7 and fulvestrant-resistant breast cancer cell lines, and the results were consistent with those of next-generation sequencing. According to the study findings, miR-148a, miR-31, miR-21, miR-498 and miR-29b were significantly upregulated, while miR-143, miR-145, miR-424 and miR-137 were significantly downregulated in fulvestrant-resistant cell lines.

Several previous studies have demonstrated that miR-143 and miR-145 are downregulated in breast cancer. For instance, miR-143 has been reported to function as a tumor suppressor in breast cancer by downregulating DNA methyltransferase 3A (DNMT3A) expression. The downregulation of miR-143 in breast cancer can lead to the overexpression of DNMT3A, which induces the hypermethylation and silencing of tumor suppressor genes, while contributing to tumorigenesis (28). A number of studies reported that the overexpression of miR-145 inhibited breast cancer cell invasion and metastasis by regulating the expression of tumor metastasis-associated suppressor genes, such as mucin 1, matrix metalloproteinase-11 and ADAM metallopeptidase domain 17, while this miRNA was also able to inhibit cell growth by targeting rhotekin and c-Myc (29-31). Our previous study also demonstrated that miR-145 overexpression induced alterations in the whole transcriptome and inhibited breast cancer development (32). Tumor suppressor miRNAs, such as miR-143 and miR-145, inhibit ERBB3 expression, and consequently suppress the proliferation and invasion of breast cancer cells (33). In the current study, the results of next-generation sequencing revealed that miR-143 and miR-145 were significantly downregulated in the three fulvestrant-resistant cell lines, which may be associated to drug resistance. Thus, it can be speculated that the interaction between miR-143/145 and their targets may serve an important role in fulvestrant resistance; however, this field requires further study.

Steroid receptor coactivator 3 (SRC3) is a SRC member of the p160 family, which is frequently amplified in breast cancer (34). miR-137 downregulates the SRC3 gene by targeting its 3'UTR, and inhibits cell proliferation and drug resistance (35). Overexpression of miR-137 in breast cancer cells can also reduce proliferation and migration by downregulating lysine demethylase 5B, estrogen-related receptor $\alpha$ and C-terminal-binding protein 1 (36-38). Zhu et al (39) reported that miR-137 was downregulated in multiple drug-resistant cell lines, including MCF-7/ADM. The overexpression of miR-137 was able to enhance the sensitivity of breast cancer cells to chemotherapeutic agents by modulating the expression of P-glycoprotein by targeting $\mathrm{Y}$ box binding protein 1 . In the present study, miR-137 was downregulated in fulvestrant-resistant cell lines, suggesting that drug sensitivity was reduced and drug resistance was enhanced. Therefore, based on these findings, it is speculated that the expression of miR-137 may alter the sensitivity of breast cancer cells to fulvestrant, and thus miR-137 may serve as a predictor of endocrine therapy.

The present study results also indicated that miR-424 expression was significantly downregulated in fulvestrant-resistant cell lines, which is consistent with the findings of a recent study (40). It has been reported that miR-424 was associated with drug resistance in breast cancer. Silencing of miR-424 was reported to activate the phosphoinositide 3-kinase/protein kinase $\mathrm{B} / \mathrm{mammalian}$ target of rapamycin (PI3K/AKT/mTOR) signaling pathway and lead to the resistance of MCF-7 cells to letrozole (41). Rodriguez-Barrueco et al (42) demonstrated that a loss of the miRNA cluster miR-424(322)/503 may induce resistance to chemotherapeutic drugs by modulating the expression levels of insulin-like growth factor 1 receptor and B-cell lymphoma 2. According to the aforementioned observations, it can be speculated that miR-424 may affect its corresponding targets and alter the signaling pathway to induce fulvestrant resistance. Thus, miR-424 may be used as a potential biomarker in the diagnosis and prognosis of fulvestrant-resistant breast cancer.

As an oncomiRNA, miR-21 has been reported to be overexpressed in numerous types of tumor, including head and neck squamous cell carcinoma, and colon and breast cancer (43). miR-21 may promote breast cancer invasion and metastasis by regulating the metastasis-associated tumor suppressor gene, tropomyosin 1 (44). Blower et al (45) reported that miR-21 was associated with the potency of anticancer agents and chemoresistance, suggesting that miR-21 may be involved in 


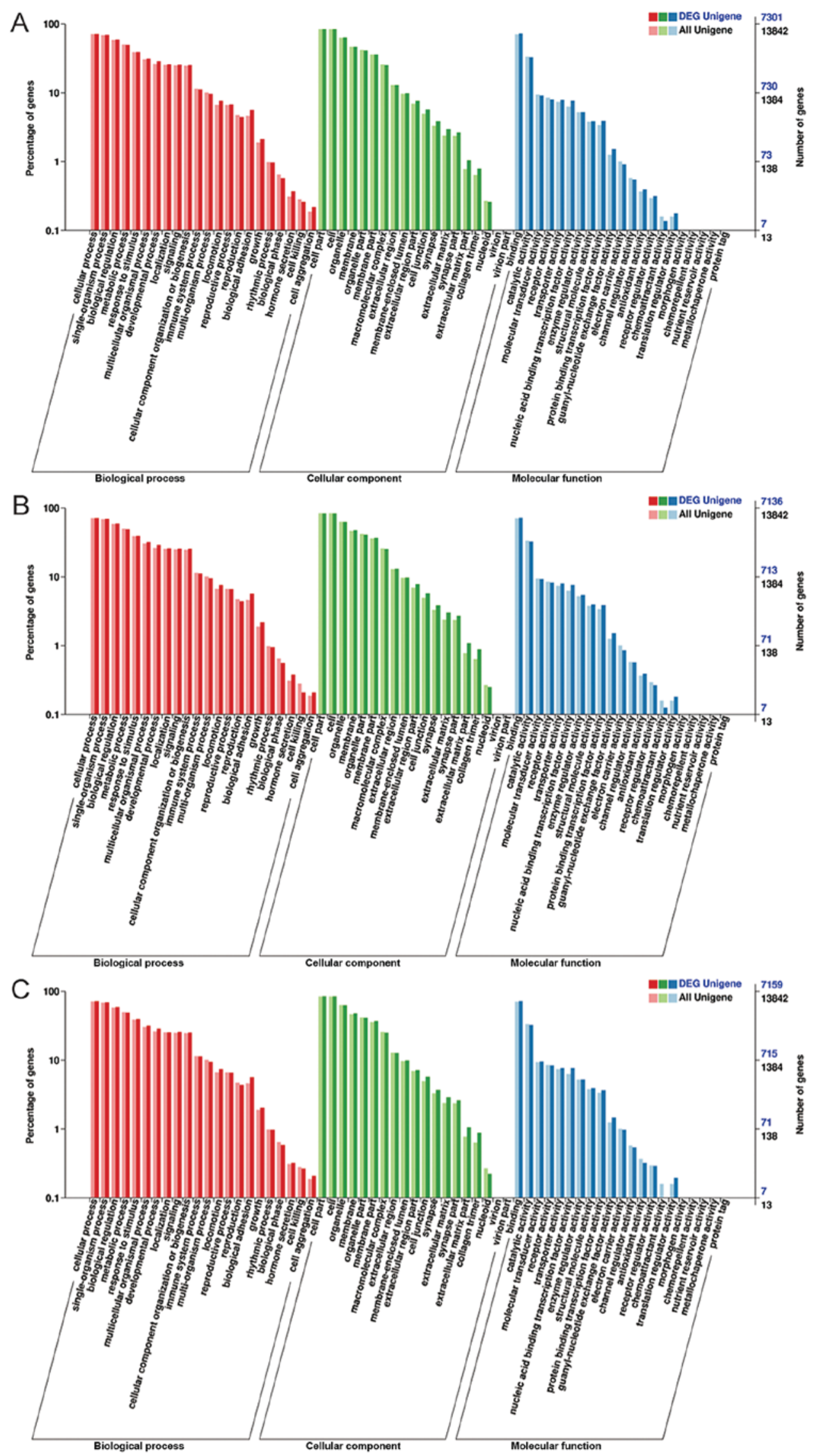

Figure 4. GO annotation analysis of differentially expressed miRNAs between the following pairs of cell lines: (A) MCF-7 vs. MCF-7-CC; (B) MCF-7 vs. MCF-7-TT; and (C) MCF-7 vs. MCF-7-21. GO classifications are displayed on the horizontal axis, while percentages of gene counts are shown on the left vertical axis and gene counts are on the right vertical axis. The enrichment of the miRNA target genes in the three GO categories (biological process, cellular component and molecular function) and secondary functions is displayed in the DEGs and in all genes. Secondary functions with a significant difference indicate the varying enrichment profile of the differentially expressed miRNA target genes and all other genes. GO, Gene Ontology; DEG, differentially expressed gene. 

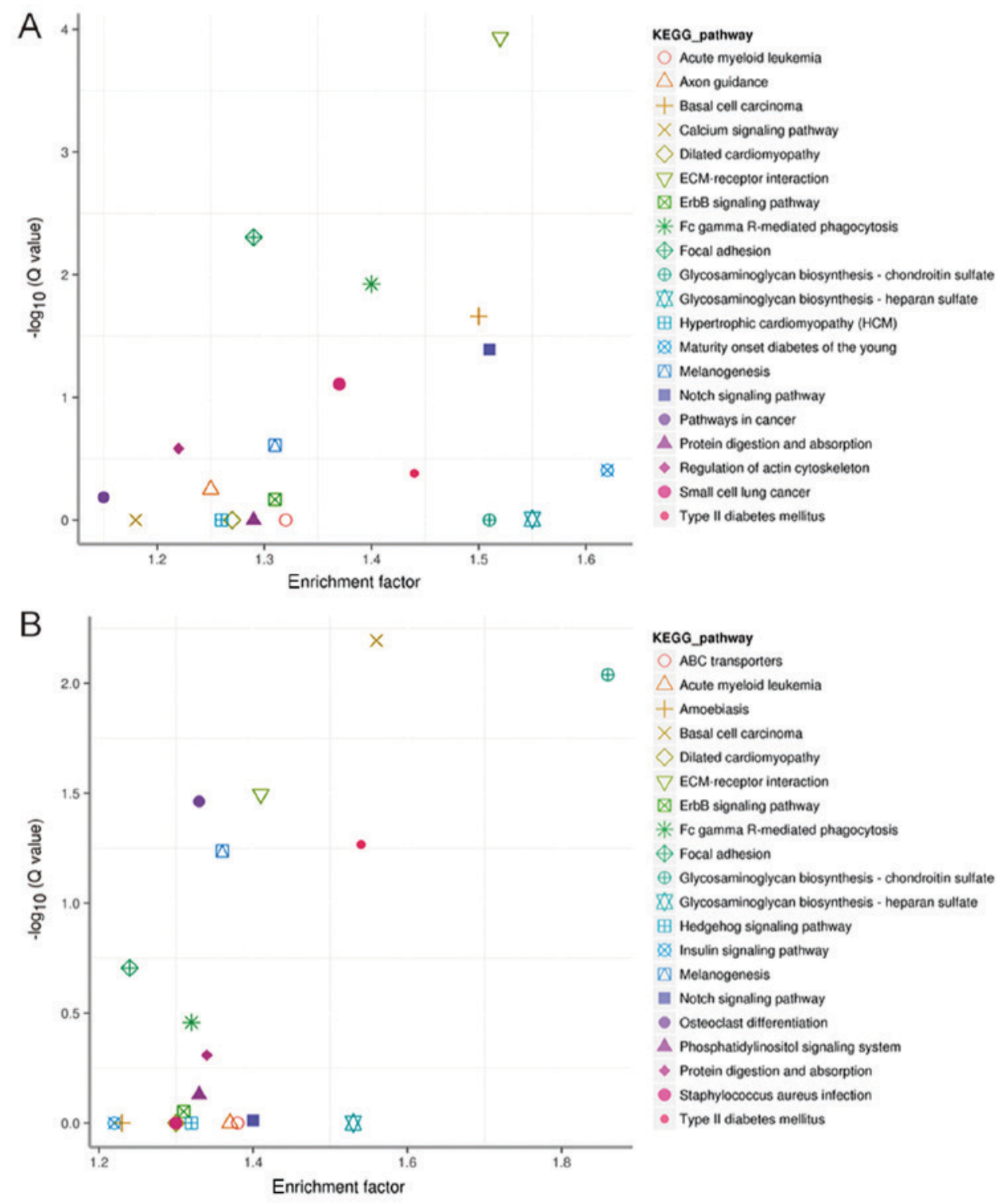

KEGG_pathway

ABC transporters

$\triangle$ Acule myeloid leukemia

+ Amoebiasis

$\times$ Basal cell carcinoma

Dilated cardiomyopathy

$\nabla$ ECM-receptor interaction

$\triangle$ ErbB signaling pathway

* Fc gamma R-mediated phagocytosis

$\oplus$ Focal adhesion

(†) Glycosaminoglycan biosynthesis - chondroitin sullate

Q8 Glycosaminogiycan biosynthesis - heparan sultate

由 Hedgehog signaling pathway

Insulin signaling pathway

Melanogenesis

notch signaling pathway

- Osteoclast difterentiation

$\Delta$ Phosphatidylinositol signaling system

- Protein digestion and absorption

Staphylococcus aureus infection

- Type II diabetes mellitus

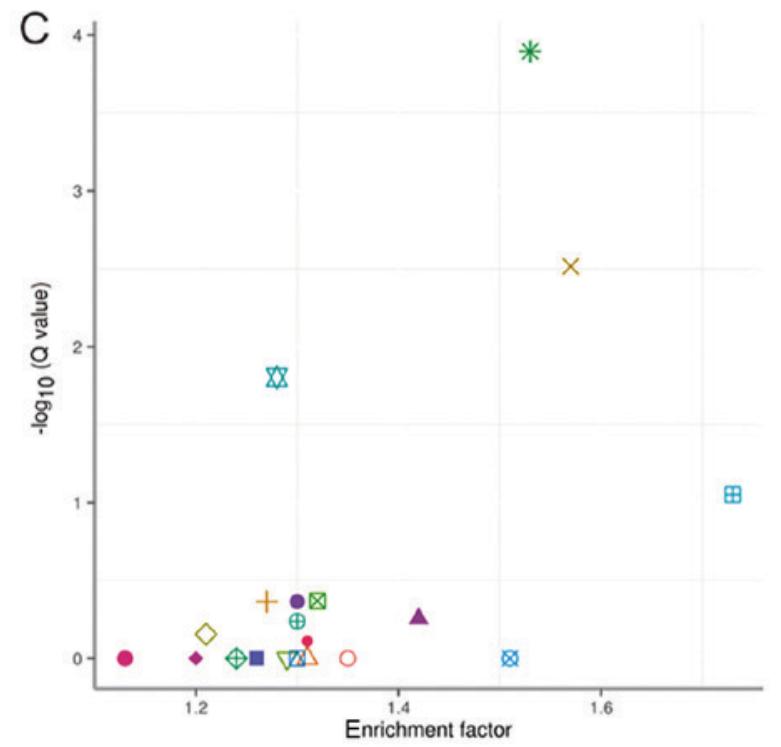

KEGG_pathway

ABC transporters

$\triangle$ Arrhythmogenic right ventricular cardiomyopathy (ARVC)

+ Axon guidance

$\times$ Basal cell carcinoma

$\checkmark$ Calcium signaling pathway

$\nabla$ Cardiac muscle contraction

$\triangle$ Dilated cardiomyopathy

* ECM-receptor interaction

$\oplus$ ErbB signaling pathway

$\oplus$ Fc gamma R-mediated phagocytosis

Focal adhesion

田 Glycosaminoglycan biosynthesis - chondroitin sulfate

Q Glycosaminoglycan biosynthesis - heparan sulfate

$\triangle$ Hedgehog signaling pathway

Eypertrophic cardiomyopathy (HCM)

- Melanogenesis

$\Delta$ Notch signaling pathway

- Osteoclast differentiation

- Pathways in cancer

- Small cell lung cancer

Figure 5. KEGG pathway analysis of differentially expressed microRNAs between the following pairs of cell lines: (A) MCF-7 vs. MCF-7-CC; (B) MCF-7 vs. MCF-7-TT; and (C) MCF-7 vs. MCF-7-21. Each shape in the graph represents a KEGG pathway, which are listed on the right. The enrichment factor shown on the horizontal axis indicates the ratio of DEGs annotated to the pathway to all genes. A greater enrichment factor indicates higher significance of the enrichment level of the DEGs in the pathway. The ordinate is $-\log _{10}(\mathrm{Q}$ value), and $\mathrm{Q}$ value is the P-value adjusted by multiple hypothesis testing. Thus, a greater ordinate indicates a more reliable significance of the enriched DEGs in the pathway. The pathways represented by different shapes in the upper right corner of the graph are of greater referential value. KEGG, Kyoto Encyclopedia of Genes and Genomes; DEG, differentially expressed gene. 
breast cancer drug resistance. It has also been demonstrated that the silencing of miR-21 confers sensitivity to tamoxifen and fulvestrant by enhancing autophagic cell death through inhibition of the PI3K/AKT/mTOR signaling pathway in breast cancer cells (46). The results of the present study indicated that miR-21 expression was increased in the MCF-7-TT cell line; thus, it is proposed that the overexpression of miR-21 may reduce the sensitivity of cells to fulvestrant and enhance drug resistance. Zhou et al (47) also reported that miR-21 may be associated with fulvestrant resistance. Estradiol was able to repress the expression of miR-21 by activating the ER in MCF-7 cells, which may explain how fulvestrant, as a pure antiestrogen agent, can increase the expression of miR-21 (48). Therefore, the interaction between Estradiol and miR-21 may be closely associated with fulvestrant resistance.

In the present study, miR-221-3p and miR-222-5p were downregulated in MCF-7-CC and MCF-7-21 cell lines, which was inconsistent with previous studies. Rao et al (9) and Xin et al (40) reported that miR-221 and miR-222 were upregulated in a fulvestrant-resistant cell line. In these studies, hormone-free medium with $10 \%$ charcoal-stripped FBS was used without phenol red to culture a fulvestrant-resistant cell line, as reported previously (14). However, the present study used DMEM (originally containing phenol red) with 5\% FBS, which has estrogenic activity (49). Thus, the different results may be due to the effects of estrogenic activity in the media used. It has been reported that medium containing charcoal-stripped serum mimics postmenopausal conditions, while unstripped serum mimics premenopausal conditions (12), suggesting that the fulvestrant-resistant cell lines obtained in the current study may exhibit cell behaviors associated with premenopausal conditions. Furthermore, ER expression levels were indicated to be markedly reduced in fulvestrant-resistant cells under premenopausal conditions, whereas almost no expression of ER was detected in fulvestrant-resistant cells under postmenopausal conditions (12). The mechanisms of resistance to estrogen antagonists differ under premenopausal and postmenopausal conditions, which may account for the inconsistencies between the present study results and those of previous studies $(9,40)$. In addition, the ER directly suppresses miR-221 and miR-222 expression in breast cancer by recruiting the corepressors nuclear receptor corepressor and silencing mediator of retinoic acid and thyroid hormone receptor (50). Further studies are required to determine the various mechanisms underlying fulvestrant resistance in premenopausal and postmenopausal conditions, and to elucidate the roles of miR-221 and miR-222 in fulvestrant resistance.

GO and KEGG pathway analyses were performed in the current study to further understand the biological function of the predicted targets and their potential roles in the development of fulvestrant resistance. Although there were a few differences in the KEGG enriched pathways among the three resistant cell lines, the results indicated that the targets of the differentially expressed miRNAs were primarily involved in ECM-receptor interaction, the Notch signaling pathway and focal adhesion. Previous studies (51-56) have demonstrated that these pathways were involved in the progression and metastasis of cancer. It has also been reported that abnormal Notch signaling may contribute to mammary carcinogenesis by deregulating the self-renewal ability of normal mammary stem cells (52). Further studies are needed to verify whether these pathways are associated with fulvestrant resistance.

The establishment of a drug-resistant cell line model in vitro may aid future investigation into the mechanism underlying drug resistance. Additionally, different methods to induce resistance are likely to result in various mechanisms of resistance. DMEM with 5\% FBS and phenol red was used to culture the fulvestrant-resistant cell lines in the present study, which may be considered to represent the patient condition in vitro (12). In the present study, three fulvestrant-resistant cell lines were established by three different methods, which may represent different drug resistance conditions. These cell lines may have exogenous or endogenous resistance characteristics, similar to the conditions of patients. Thus, these different fulvestrant-resistant cell lines were used to investigate the association between differential miRNA expression profiles and fulvestrant resistance of human breast cancer cell lines. The MCF-7-CC cell line was established by stepwise induction, starting with low concentrations of fulvestrant. Following treatment with the corresponding concentration of fulvestrant screening medium, replacement of the drug-free complete medium, removal of apoptotic cells and proliferation of viable cells, tumor cells were allowed an appropriate buffer time to activate certain physiological pathways and adapt to stimulation with the drug in order to acquire resistance. It has been reported the induction of a drug-resistant cell line in a stepwise manner resulted in alterations in the physiology, genetics and remodeling, and cells acquired resistance during the process of screening and culturing $(57,58)$. Therefore, it can be speculated that the MCF-7-CC cell line established in the current study may be an acquired drug-resistant cell line. For the drug-resistant cell line MCF-7-TT, which was established in a stepwise temporal manner starting with a high concentration of fulvestrant, the induction time increased gradually from 1 to $48 \mathrm{~h}$, and then to $72 \mathrm{~h}$. During the induction, apoptotic cells were removed and viable cells proliferated, which provided a buffer time for repair of slightly damaged cells and eliminate relatively drug sensitive and severely damaged cells; however, cells with inherent relatively resistant properties were maintained. Yang and Trujillo (59) reported that discontinuous screening and cultivating resistant cells with high concentrations is mainly conducted by inducing the death of sensitive cells with high concentrations of drugs and maintaining those with inherently drug-resistant properties. Therefore, it is speculated that the MCF-7-TT cell line obtained in the current study may arise from a combination of exogenous and endogenous resistance-associated factors. Finally, for the drug-resistant cell line MCF-7-21, drug resistance was established by incubating the cells with a high concentration of fulvestrant for 21 days; apoptotic cells were not removed, while viable cells did not proliferate during the induction. As a period of time for cells to adapt to treatment with fulvestrant was not provided, it is speculated that MCF-7-21 may be an endogenous resistant cell line. However, it was difficult to distinguish between the endogenous and acquired forms of resistance. The differential miRNA expression profiles varied among the three fulvestrant-resistant cell lines, suggesting that the drug-resistant cell lines may undertake different molecular mechanisms of 
fulvestrant resistance. Therefore, further study is required to elucidate the association among these mechanisms.

In conclusion, the current study investigated the association between differential miRNA expression profiles and fulvestrant resistance in human breast cancer cells. A number of differentially expressed miRNAs were identified in the present study. The current results suggest that miR-143, miR-145, miR-424, miR-137 and miR-21 may serve important roles in fulvestrant resistance in breast cancer and may be potential targets for breast cancer treatment. As there are few studies regarding the mechanisms of miRNAs in fulvestrant resistance, the present study may provide the basis for future research into fulvestrant resistance and development of new strategies for the treatment of patients with fulvestrant-resistant breast cancer. The functional analysis of miRNAs in fulvestrant resistance will also serve an important role in the treatment of drug-resistant breast cancer. According to the results of this preliminary study, one or several differentially expressed miRNAs of interest will be selected in our further studies, which will investigate the association between miRNAs and fulvestrant resistance.

\section{Acknowledgements}

Not applicable.

\section{Funding}

The present study was supported by grants from the National Natural Science Foundation of China (nos. 81272372 and 30873044) and the Zhongnan Hospital of Wuhan University Science, Technology and Innovation Seed Fund (grant no. znpy2016033). The project was also sponsored by the Scientific Research Foundation for Returned Overseas Chinese Scholars, State Education Ministry.

\section{Availability of data and materials}

The datasets used and/or analyzed during the current study are available from the corresponding author on reasonable request.

\section{Authors' contributions}

$\mathrm{XL}, \mathrm{KH}$ and $\mathrm{HZ}$ designed the study. $\mathrm{KH}$ and $\mathrm{HZ}$ performed the experiments; JG, KH, HZ, YS, PY, QZ and ZP collected and analyzed the data. XL, JG, KH and HZ wrote the manuscript. All authors read and approved the final manuscript.

\section{Ethics approval and consent to participate}

Not applicable.

\section{Patient consent for publication}

Not applicable.

\section{Competing interests}

The authors declare that they have no competing interests.

\section{References}

1. Siegel RL, Miller KD and Jemal A: Cancer statistics, 2016. CA Cancer J Clin 66: 7-30, 2016.

2. Osborne CK, Wakeling A and Nicholson RI: Fulvestrant: An oestrogen receptor antagonist with a novel mechanism of action. Br J Cancer 90 (Suppl 1): S2-S6, 2004.

3. Tangkeangsirisin W and Serrero G: GP88 (Progranulin) confers fulvestrant (Faslodex, ICI 182,780) resistance to human breast cancer cells. Adv Breast Cancer Res 03: 68-78, 2014.

4. Osipo C, Meeke K, Cheng D, Weichel A, Bertucci A, Liu H and Jordan VC: Role for HER2/neu and HER3 in fulvestrant-resistant breast cancer. Int J Oncol 30: 509-520, 2007.

5. Tsuboi K, Kaneko Y, Nagatomo T, Fujii R, Hanamura T, Gohno T, Yamaguchi Y, Niwa T and Hayashi SI: Different epigenetic mechanisms of ERalpha implicated in the fate of fulvestrant-resistant breast cancer. J Steroid Biochem Mol Biol 167: 115-125, 2017.

6. Hong L, Yang Z, Ma J and Fan D: Function of miRNA in controlling drug resistance of human cancers. Curr Drug Targets 14: $1118-1127,2013$.

7. Brennecke J, Hipfner DR, Stark A, Russell RB and Cohen SM: bantam encodes a developmentally regulated microRNA that controls cell proliferation and regulates the proapoptotic gene hid in Drosophila. Cell 113: 25-36, 2003.

8. Inui M, Martello G and Piccolo S: MicroRNA control of signal transduction. Nat Rev Mol Cell Biol 11: 252-263, 2010.

9. Rao X, Di Leva G, Li M, Fang F, Devlin C, Hartman-Frey C, Burow ME, Ivan M, Croce CM and Nephew KP: MicroRNA-221/222 confers breast cancer fulvestrant resistance by regulating multiple signaling pathways. Oncogene 30 : 1082-1097, 2011.

10. Ji S, Shao G, Lv X, Liu Y, Fan Y, Wu A and Hu H: Downregulation of miRNA-128 sensitises breast cancer cell to chemodrugs by targeting Bax. Cell Biol Int 37: 653-658, 2013.

11. Hu Q, Gong JP, Li J, Zhong SL, Chen WX, Zhang JY, Ma TF, Ji H, Lv MM, Zhao JH and Tang JH: Down-regulation of miRNA-452 is associated with adriamycin-resistance in breast cancer cells. Asian Pac J Cancer Prev 15: 5137-5142, 2014.

12. Fan P, Yue W, Wang JP, Aiyar S, Li Y, Kim TH and Santen RJ: Mechanisms of resistance to structurally diverse antiestrogens differ under premenopausal and postmenopausal conditions: Evidence from in vitro breast cancer cell models. Endocrinology 150: 2036-2045, 2009.

13. Ye P, Fang C, Zeng H, Shi Y, Pan Z, An N, He K, Zhang L and Long $X$ : Differential microRNA expression profiles in tamoxifen-resistant human breast cancer cell lines induced by two methods. Oncol Lett 15: 3532-3539, 2018.

14. Fan M, Yan PS, Hartman-Frey C, Chen L, Paik H, Oyer SL, Salisbury JD, Cheng AS, Li L, Abbosh PH, et al: Diverse gene expression and DNA methylation profiles correlate with differential adaptation of breast cancer cells to the antiestrogens tamoxifen and fulvestrant. Cancer Res 66: 11954-11966, 2006.

15. Jensen BL, Skouv J, Lundholt BK and Lykkesfeldt AE: Differential regulation of specific genes in MCF-7 and the ICI 182780-resistant cell line MCF-7/182R-6. Br J Cancer 79: 386-392, 1999.

16. Coser KR, Wittner BS, Rosenthal NF, Collins SC, Melas A, Smith SL, Mahoney CJ, Shioda K, Isselbacher KJ, Ramaswamy S and Shioda T: Antiestrogen-resistant subclones of MCF-7 human breast cancer cells are derived from a common monoclonal drug-resistant progenitor. Proc Natl Acad Sci USA 106: 14536-14541, 2009.

17. Stokowy T, Eszlinger M, Świerniak M, Fujarewicz K, Jarząb B, Paschke R and Krohn K: Analysis options for high-throughput sequencing in miRNA expression profiling. BMC Res Notes 7: $144,2014$.

18. Friedländer MR, Mackowiak SD, Li N, Chen W and Rajewsky N: miRDeep2 accurately identifies known and hundreds of novel microRNA genes in seven animal clades. Nucleic Acids Res 40: 37-52, 2012.

19. Fahlgren N, Howell MD, Kasschau KD, Chapman EJ, Sullivan CM, Cumbie JS, Givan SA, Law TF, Grant SR, Dangl JL and Carrington JC: High-throughput sequencing of Arabidopsis microRNAs: Evidence for frequent birth and death of MIRNA genes. PLoS One 2: e219, 2007.

20. Li J, Witten DM, Johnstone IM and Tibshirani R: Normalization, testing, and false discovery rate estimation for RNA-sequencing data. Biostatistics 13: 523-538, 2012. 
21. Deng W, Wang Y, Liu Z, Cheng H and Xue Y: HemI: A toolkit for illustrating heatmaps. PLoS One 9: e111988, 2014.

22. Livak KJ and Schmittgen TD: Analysis of relative gene expression data using real-time quantitative PCR and the 2(-Delta Delta C(T)) method. Methods 25: 402-408, 2001.

23. Betel D, Wilson M, Gabow A, Marks DS and Sander C: The microRNA.org resource: Targets and expression. Nucleic Acids Res 36: D149-D153, 2008.

24. Rehmsmeier M, Steffen P, Hochsmann M and Giegerich R: Fast and effective prediction of microRNA/target duplexes. RNA 10 : $1507-1517,2004$

25. Young MD, Wakefield MJ, Smyth GK and Oshlack A: Gene ontology analysis for RNA-seq: Accounting for selection bias. Genome Biol 11: R14, 2010.

26. Mao X, Cai T, Olyarchuk JG and Wei L: Automated genome annotation and pathway identification using the KEGG Orthology (KO) as a controlled vocabulary. Bioinformatics 21 : 3787-3793, 2005.

27. Zhang B, Pan X, Cobb GP and Anderson TA: microRNAs as oncogenes and tumor suppressors. Dev Biol 302: 1-12, 2007.

28. Ng EK, Li R, Shin VY, Siu JM, Ma ES and Kwong A: MicroRNA-143 is downregulated in breast cancer and regulates DNA methyltransferases $3 \mathrm{~A}$ in breast cancer cells. Tumour Biol 35: 2591-2598, 2014

29. Sachdeva M and Mo YY: miR-145-mediated suppression of cell growth, invasion and metastasis. Am J Transl Res 2: 170-180, 2010.

30. Wang S, Bian C, Yang Z, Bo Y, Li J, Zeng L, Zhou H and Zhao RC: miR-145 inhibits breast cancer cell growth through RTKN. Int J Oncol 34: 1461-1466, 2009.

31. Sachdeva M, Zhu S, Wu F, Wu H, Walia V, Kumar S, Elble R, Watabe $\mathrm{K}$ and Mo YY: p53 represses c-Myc through induction of the tumor suppressor miR-145. Proc Natl Acad Sci USA 106 3207-3212, 2009.

32. Ye P, Shi Y, An N, Zhou Q, Guo J and Long X: miR-145 overexpression triggers alteration of the whole transcriptome and inhibits breast cancer development. Biomed Pharmacother 100 72-82, 2018.

33. Yan X, Chen X, Liang H, Deng T, Chen W, Zhang S, Liu M, Gao X, Liu Y, Zhao C, et al: miR-143 and miR-145 synergistically regulate ERBB3 to suppress cell proliferation and invasion in breast cancer. Mol Cancer 13: 220, 2014.

34. Gojis O, Rudraraju B, Gudi M, Hogben K, Sousha S, Coombes RC Cleator S and Palmieri C: The role of SRC-3 in human breast cancer. Nat Rev Clin Oncol 7: 83-89, 2010.

35. Eedunuri VK, Rajapakshe K, Fiskus W, Geng C, Chew SA Foley C, Shah SS, Shou J, Mohamed JS, Coarfa C, et al: miR-137 targets p160 steroid receptor coactivators SRC1, SRC2, and SRC3 and inhibits cell proliferation. Mol Endocrinol 29: 1170-1183, 2015.

36. Denis H, Van Grembergen O, Delatte B, Dedeurwaerder S, Putmans P, Calonne E, Rothé F, Sotiriou C, Fuks F and Deplus R: MicroRNAs regulate KDM5 histone demethylases in breast cancer cells. Mol Biosyst 12: 404-413, 2016.

37. Zhao Y, Li Y, Lou G, Zhao L, Xu Z, Zhang Y and He F: MiR-137 targets estrogen-related receptor alpha and impairs the proliferative and migratory capacity of breast cancer cells. PLoS One 7 e39102, 2012.

38. Han Y, Bi Y, Bi H, Diao C, Zhang G, Cheng K and Yang Z: miR-137 suppresses the invasion and procedure of EMT of human breast cancer cell line MCF-7 through targeting CtBP1. Hum Cell 29: 30-36, 2016.

39. Zhu X, Li Y, Shen H, Li H, Long L, Hui L and Xu W: miR-137 restoration sensitizes multidrug-resistant MCF-7/ADM cells to anticancer agents by targeting YB-1. Acta Biochim Biophys Sin (Shanghai) 45: 80-86, 2013 .

40. Xin F, Li M, Balch C, Thomson M, Fan M, Liu Y, Hammond SM, Kim S and Nephew KP: Computational analysis of microRNA profiles and their target genes suggests significant involvement in breast cancer antiestrogen resistance. Bioinformatics 25 430-434, 2009

41. Vilquin P, Donini CF, Villedieu M, Grisard E, Corbo L, Bachelot T, Vendrell JA and Cohen PA: MicroRNA-125b upregulation confers aromatase inhibitor resistance and is a novel marker of poor prognosis in breast cancer. Breast Cancer Res 17: 13,2015.
42. Rodriguez-Barrueco R, Nekritz EA, Bertucci F, Yu J Sanchez-Garcia F, Zeleke TZ, Gorbatenko A, Birnbaum D, Ezhkova E, Cordon-Cardo C, et al: miR-424(322)/503 is a breast cancer tumor suppressor whose loss promotes resistance to chemotherapy. Genes Dev 31: 553-566, 2017.

43. Fu X, Han Y, Wu Y, Zhu X, Lu X, Mao F, Wang X, He X, Zhao Y and Zhao Y: Prognostic role of microRNA-21 in various carcinomas: A systematic review and meta-analysis. Eur J Clin Invest 41: 1245-1253, 2011.

44. Zhu S, Wu H, Wu F, Nie D, Sheng S and Mo YY: MicroRNA-21 targets tumor suppressor genes in invasion and metastasis. Cell Res 18: 350-359, 2008

45. Blower PE, Chung JH, Verducci JS, Lin S, Park JK, Dai Z, Liu CG, Schmittgen TD, Reinhold WC, Croce CM, et al: MicroRNAs modulate the chemosensitivity of tumor cells. Mol Cancer Ther 7: 1-9, 2008

46. Yu X, Li R, Shi W, Jiang T, Wang Y, Li C and Qu X: Silencing of microRNA-21 confers the sensitivity to tamoxifen and fulvestrant by enhancing autophagic cell death through inhibition of the PI3K-AKT-mTOR pathway in breast cancer cells. Biomed Pharmacother 77: 37-44, 2016.

47. Zhou Q, Zeng H, Ye P, Shi Y, Guo J and Long X: Differential microRNA profiles between fulvestrant-resistant and tamoxifen-resistant human breast cancer cells. Anticancer Drugs 29: 539-548, 2018.

48. Wickramasinghe NS, Manavalan TT, Dougherty SM, Riggs KA, Li Y and Klinge CM: Estradiol downregulates miR-21 expression and increases miR-21 target gene expression in MCF-7 breast cancer cells. Nucleic Acids Res 37: 2584-2595, 2009.

49. Welshons WV, Wolf MF, Murphy CS and Jordan VC: Estrogenic activity of phenol red. Mol Cell Endocrinol 57: 169-178, 1988.

50. Di Leva G, Gasparini P, Piovan C, Ngankeu A, Garofalo M, Taccioli C, Iorio MV, Li M, Volinia S, Alder H, et al: MicroRNA cluster 221-222 and estrogen receptor alpha interactions in breast cancer. J Natl Cancer Inst 102: 706-721, 2010.

51. Yeh MH, Tzeng YJ, Fu TY, You JJ, Chang HT, Ger LP and Tsai KW: Extracellular matrix-receptor interaction signaling genes associated with inferior breast cancer survival. Anticancer Res 38: 4593-4605, 2018

52. Dontu G, Jackson KW, McNicholas E, Kawamura MJ, Abdallah WM and Wicha MS: Role of Notch signaling in cell-fate determination of human mammary stem/progenitor cells. Breast Cancer Res 6: R605-R615, 2004.

53. Li L, Tang P, Li S, Qin X, Yang H, Wu C and Liu Y: Notch signaling pathway networks in cancer metastasis: A new target for cancer therapy. Med Oncol 34: 180, 2017.

54. Alketbi A and Attoub S: Notch signaling in cancer: Rationale and strategies for targeting. Curr Cancer Drug Targets 15: 364-374, 2015.

55. Hoskin V, Szeto A, Ghaffari A, Greer PA, Cote GP and Elliott BE: Ezrin regulates focal adhesion and invadopodia dynamics by altering calpain activity to promote breast cancer cell invasion. Mol Biol Cell 26: 3464-3479, 2015

56. Eke I and Cordes N: Focal adhesion signaling and therapy resistance in cancer. Semin Cancer Biol 31: 65-75, 2015.

57. Negoro K, Yamano Y, Fushimi K, Saito K, Nakatani K, Shiiba M, Yokoe H, Bukawa H, Uzawa K, Wada T, et al: Establishment and characterization of a cisplatin-resistant cell line, KB-R, derived from oral carcinoma cell line, KB. Int J Oncol 30: 1325-1332, 2007.

58. Watson MB, Lind MJ and Cawkwell L: Establishment of in-vitro models of chemotherapy resistance. Anticancer Drugs 18: 749-754, 2007.

59. Yang LY and Trujillo JM: Biological characterization of multidrug-resistant human colon carcinoma sublines induced/selected by two methods. Cancer Res 50: 3218-3225, 1990.

This work is licensed under a Creative Commons Attribution-NonCommercial-NoDerivatives 4.0 International (CC BY-NC-ND 4.0) License. 